\title{
Interface damage model for fatigue-driven fracture
}

\author{
Feiyu Geng*, Akke S. J. Suiker \\ Department of the Built Environment, Eindhoven University of Technology, P.O. box 513, 5600 MB Eindhoven, The \\ Netherlands
}

\begin{abstract}
A fatigue interface damage model is presented that is based on combining a fatigue evolution law with a static interface damage model. The fatigue model elegantly enables the simulation of crack initiation and propagation in a computationally efficient and accurate way, accounting for mixed-mode loading conditions and fatigue loading of variable amplitude. The main features of the fatigue model are explained and demonstrated by means of an illustrative numerical example.
\end{abstract}

\section{Introduction}

Cohesive zone models have been widely used to numerically predict discrete cracking phenomena in brittle and ductile materials subjected to static loading conditions, see for example, [1-4]. At the crack tip of the cohesive zone the traction across the crack surfaces is related to the relative displacement of the crack faces (= crack face separation) by means of a traction-separation law. Accordingly, the ultimate traction determines the critical stress level at which the crack nucleates, and the area under the traction-separation law, which equals the fracture toughness, characterizes the propagation characteristics of the crack.

In the literature various suggestions have been made to extend static cohesive zone models towards the case of fatigue. When based on Paris law, this requires the calculation of the crack driving force at the crack tip, which corresponds to the energy released per unit advance of crack length, see [5-8]. The crack driving force can be straightforwardly established for a single crack with a well-defined crack tip, but cannot be uniquely computed in case of complex fracture patterns characterized by (various) crack coalescence and crack bifurcation events. In order to circumvent this problem, various investigators have defined the fatigue growth rate as a function of the local deformation in a material point of the crack [9-12]. In the approach of Khoramishad et al. [9,12], the resulting fatigue damage parameter degrades the static traction-separation diagram that is used for computing the traction and the effective damage under the actual cyclic loading conditions applied. This local approach for the computation of the strength degradation during fatigue has the advantage that complex fracture patterns composed of multiple cracks can be analyzed in a natural and robust fashion.

Inspired by the approach of Khoramishad et al. $[9,12]$, this paper presents a fatigue interface damage model that is based on combining the fatigue evolution law presented in [12] with the static interface damage model presented in [1]. The fatigue model elegantly enables the simulation of crack initiation and propagation in a computationally efficient and accurate way, accounting for mixed-mode loading conditions and fatigue loading of variable amplitude.

\section{Fatigue interface damage model}

The interface damage model used for simulating fracture under fatigue loading is constructed by combining the fatigue damage law suggested in [12] with the static interface damage model proposed in [1]. The combination of the two models is described below, followed by listing the main features of the fatigue interface damage model.

\subsection{Review of the static interface damage model}

In the static interface damage model proposed in [1] the cohesive tractions $\boldsymbol{t}$ in an interface material point are related to the effective relative displacements $\boldsymbol{v}$ as

$$
t_{i}=(1-D) K v_{i}-D K \delta_{1 i}\left\langle-v_{1}\right\rangle \text { where } i \in\{1,2\},
$$

with the indices ' 1 ' and ' 2 ' denoting the components in normal and shear directions, respectively. Further, $K$ is the elastic stiffness (with dimension of force $\times$ length $^{-3}$ ), and $\delta_{i j}$ is the Kronecker delta symbol. Note that the Macauley brackets $\langle x\rangle=(x+|x|)$ are used to avoid interpenetration of the crack faces when $v_{1}$ is negative. The damage parameter $D=0$ refers to an undamaged state and $D=1$ represents the fully damaged state of a material point. A typical bi-linear traction-separation law, schematized in Fig. 1, is adopted to specify the softening behavior experienced during damage. Here, $\kappa$ is a history parameter, whereby the onset of damage relates to $\kappa=v^{0}$, and completion of damage is reflected by $\kappa=v^{u}$.

\footnotetext{
Corresponding author: f.geng@tue.nl
} 


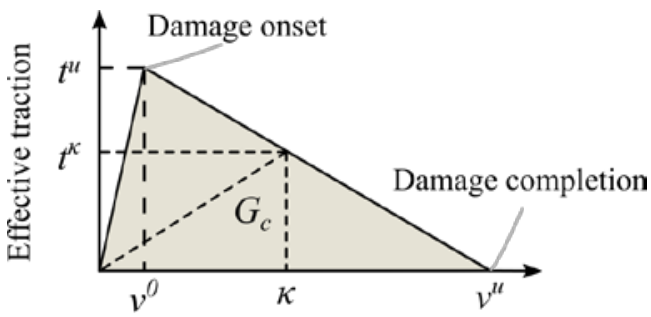

Effective relative displacement

Fig. 1. A bi-linear traction-separation law under static loading (taken from [1]).

The evolution of the damage parameter $D=\widehat{D}(\kappa)$ occurs in accordance with the rate-dependent kinetic law

$$
\dot{D}=\left\{\begin{array}{cc}
\frac{\hat{F}(\lambda, \kappa)}{\eta} & \text { for } \lambda \geq \kappa \text { and } v^{0} \leq \kappa<v^{u} \\
0 & \text { for } 0 \leq \lambda<\kappa \text { or } \kappa=v^{u}
\end{array},\right.
$$

where $\lambda$ is the effective relative (crack face) displacement $\lambda=|| \mathbf{v}||=\sqrt{v_{1}^{2}+v_{2}^{2}}$, and $\eta$ is a relaxation parameter characterizing the rate dependency of the damage process. Further, the damage loading function has the form

$$
\hat{F}(\lambda, \kappa)=\hat{f}(\lambda)-\widehat{D}(\kappa)=\frac{v^{u}\left(\lambda-v^{0}\right)}{\lambda\left(v^{u}-v^{0}\right)}-\frac{v^{u}\left(\kappa-v^{0}\right)}{\kappa\left(v^{u}-v^{0}\right)} .
$$

The upper expression in Eq.(2) reflects the damage rate when the effective relative displacement exceeds the damage history variable $\kappa$, with $v^{0} \leq \kappa<v^{u}$; the lower expression sets the damage rate to be zero when $0 \leq \lambda<\kappa$, which corresponds to: (i) the threshold value for damage nucleation has not (yet) been reached, or (ii) the material point is experiencing unloading or (iii) the material point has been fully damaged.

To simulate fracture processes under arbitrary loading conditions, a mode-mixity parameter is defined as

$$
\beta=\frac{\left|v_{2}\right|}{\left|v_{2}\right|+\left\langle v_{1}\right\rangle},
$$

with $\beta=0$ for pure mode I loading conditions $\left(v_{2}=0\right)$ and $\beta=1$ for pure mode II loading conditions $\left(v_{1}=0\right)$. By starting from a common energy-based, mixed-mode failure criterion [13]

$$
\frac{G_{I}}{G_{I, c}}+\frac{G_{I I}}{G_{I I, c}}=\frac{G}{G_{c}}
$$

where $G_{I}, G_{I I}$ and $G$ are the mode I, mode II and total energy release rates and $G_{I, c}, G_{I I, c}$ and $G_{c}$ are the corresponding critical values, i.e., the fracture toughnesses, the effective displacement at damage onset and damage completion can be, respectively, derived as [1]:

$$
v^{0}=\hat{v}^{0}(\beta)=v_{1}^{0} v_{2}^{0} \sqrt{\frac{1+2 \beta^{2}-2 \beta}{\left(\beta v_{1}^{0}\right)^{2}+\left((1-\beta) v_{2}^{0}\right)^{2}}},
$$

and

$$
v^{u}=\hat{v}^{u}(\beta)=\frac{2\left(1+2 \beta^{2}-2 \beta\right)}{K v^{0}}\left[\left(\frac{(1-\beta)^{2}}{G_{I, C}}\right)+\left(\frac{\beta^{2}}{G_{I I, c}}\right)\right]^{-1} .
$$

Here, $v_{1}^{0}=t_{1}^{u} / K$ and $v_{2}^{0}=t_{2}^{u} / K$ are the effective displacements at which damage nucleates under pure mode I and pure mode II loading conditions, respectively, with $t_{1}^{u}$ and $t_{2}^{u}$ the ultimate tractions in mode I and mode II, respectively.

\subsection{Fatigue formulation}

\subsubsection{Fatigue evolution law}

In the present section the static interface damage model above is extended towards a fatigue formulation. For his purpose, a fatigue damage parameter $D_{f}$ is introduced, which incrementally evolves by $\Delta D_{f}$ under a load cycle increment $\Delta N$ as [12]:

$$
\frac{\Delta D_{f}}{\Delta N}=\left\{\begin{array}{cl}
\alpha\left[\left(\lambda_{\max }-\lambda_{t h}\right) \gamma^{n}\right]^{\mathrm{p}} & \text { for } \lambda_{\max }>\lambda_{t h} \\
0 & \text { for } \lambda_{\max } \leq \lambda_{t h}
\end{array},\right.
$$

with the load factor $\gamma$ given by

$$
\gamma=\frac{(1-R) / 2}{1-\left[\frac{P_{\max }}{2 P_{S}}(1+R)\right]^{m}} .
$$

Here, the load ratio is given by $R=P_{\text {min }} / P_{\text {max }}$, with $P_{\min }$ the minimum amplitude of the fatigue loading, $P_{\text {max }}$ is the maximum amplitude of the fatigue loading, and $P_{S}$ the ultimate static strength obtained from a preliminary static analysis. Further, $\alpha, m, n$ and $p$ are calibration parameters, and $\lambda_{\max }$ and $\lambda_{t h}$ are the maximum effective displacement and the corresponding threshold value above which fatigue is activated, respectively, with $\lambda_{\max }$ given by

$$
\lambda_{\max }=\frac{v_{1}}{2}+\sqrt{\left(\frac{v_{1}}{2}\right)^{2}+\left(\frac{v_{2}}{2}\right)^{2}},
$$

where $v_{1}$ and $v_{2}$ are the relative crack face displacements in the normal and shear directions, respectively.

Similar to the damage parameter $D$ introduced in Section 2.1, the fatigue damage parameter $D_{f}$ is bounded as $0 \leq D_{f} \leq 1$. As shown by Eqs.(8) and (10), the evolution of $D_{f}$ is set by the local, relative crack face displacements in a material point of the crack. The upper expression in Eq.(8) represents the incremental change of the fatigue damage parameter when the effective deformation surpasses the threshold value; otherwise, the incremental fatigue damage is set to zero, as indicated by the lower expression in Eq.(8).

\subsubsection{Degrading traction-separation law}

Fig. 2 illustrates the scheme used for combining the static interface damage model reviewed in Section 2.1 with the fatigue damage evolution law, Eq.(8). The procedure essentially consists of two steps:

(a) In the first step the maximum amplitude of the fatigue loading, $P_{\max }$, is quasi-statically imposed on the actual structure in a load-controlled fashion. The possible cracking generated under this loading is 
computed with the static interface damage model outlined in Section 2.1.

(b) In the second step cracking can (further) develop, but now in a load-cycle driven fashion. Accordingly, a number of load cycles $\Delta N$ is applied incrementally, which, via Eq.(8), results in incremental fatigue damage $\Delta D_{f}$ if the effective relative separation $\lambda_{\max }$ computed in the first, quasi-static loading step is larger than the threshold value $\lambda_{t h}$. The subsequent incremental update $D_{f}$ of the fatigue damage parameter is used to reduce the strength and toughness parameters defining the tractionseparation law of the static interface damage model, in accordance with

$$
\zeta_{i}=\left(1-D_{f}\right) \zeta_{i}^{0} \text { where } i \in\{1,2\}
$$

with $\zeta_{i}$ representing the normal (index "1") and shear (index "2") components of the strength and toughness parameters, given by $t_{1}^{u}, t_{2}^{u}, G_{I, c}$ and $G_{I I, c}$. The initial values $\zeta_{i}^{0}$ of these parameters are represented by $t_{1}^{u 0}, t_{2}^{u 0}, G_{I, C}^{0}$ and $G_{I I, C}^{0}$. In this way, the fatigue damage parameter $D_{f}$ effectively degrades the static tractionseparation law. From the degraded traction-separation law the traction is subsequently computed using the static interface damage model outlined in Section 2.1. This update procedure is iteratively repeated until the mechanical system has converged towards equilibrium, after which a new load cycle increment $\Delta N$ is imposed and step 2 is repeated.

The ultimate separation of a material point in a crack is reached either when the effective damage parameter $D$ reaches unity, $D=1$, or when the fatigue damage parameter reaches unity $D_{f}=1$. Note that $D=1$ can be reached when (i) $D_{f}=1$ or (ii) $0<D_{f}<1$.

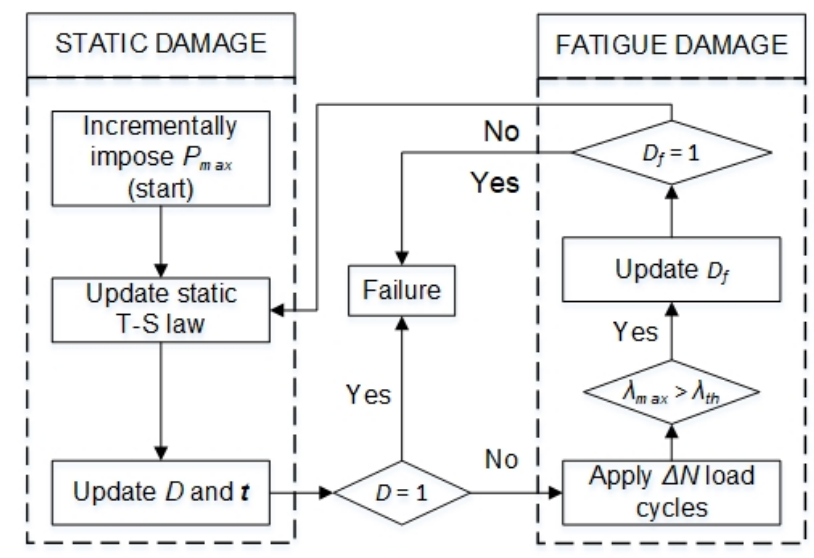

Fig. 2. The computational scheme for a fatigue simulation, whereby the static interface damage model outlined in Section 2.1 is combined with the fatigue damage evolution law, Eq.(8). $\mathrm{T}-\mathrm{S}$ denotes the traction-separation law.

It is instructive to consider the sequence of fatigue damage initiation and propagation in an interfacial crack generated in a double-cantilever beam subjected to pure model I (tensile) loading, as shown in Fig. 3. Specific material points in the interface of the double-cantilever beam specimen are indicated by circle, square and cross symbols. The material point indicated by a circle is considered to be the reference material point, which successively experiences three different types of response, as explained below.

(a) Initially the amplitude of the fatigue loading is applied in a quasi-static fashion, see Fig. 3a, whereby the response in the interface of the double-cantilever beam specimen follows the static interface damage model presented in Section 2.1. Observe that the reference material point indicated by a circle is in the elastic regime. Conversely, the material point marked with a square already experiences damage development, whereby its response lies on the softening curve. The material point marked with a cross has completely damaged, and the crack faces at this material point have fully separated.

(b) Once the fatigue amplitude has been imposed, the process becomes driven by load cycles, see Fig. 3b. The number of load cycles incrementally applied equals $\Delta N=N 1$, which will induce fatigue cracking in the interfacial material point if the corresponding value $\lambda_{\max }$ exceeds the threshold $\lambda_{t h}$. The fatigue damage reduces the strength and toughness in accordance with Eq.(11), leading to the degraded traction-separation diagram indicated in the middle figure of Fig. $3 \mathrm{~b}$. The reference material point indicated by the circle now experiences damage, whereby both the static damage parameter and the fatigue damage parameter lie between zero and one, i.e. $0<D<1$ and $0<D_{f}<1$. The material point marked by the square symbol has fully damaged, with $D=1$ and $0<D_{f}<1$.

(c) The load cycle process is continued by applying a new load cycle increment, $\Delta N=N 2-N 1$, as shown in Fig. 3c. Due to this new load cycle increment the strength and toughness have decreased in accordance with Eq.(8), as a result of which the traction-separation law has further degraded. Accordingly, the reference material point approaches the fully damaged state, whereby the relative separation of the crack faces is close to the ultimate separation $v^{u}$.

\subsubsection{Specific features of fatigue model}

Some specific features of the proposed fatigue interface damage model are summarized below:

- Arbitrary cracking paths can be simulated by placing interface elements in between the continuum elements modeling the bulk response of the structure, in accordance with the approach originally advocated in [14]. This naturally enables the simulation of fatigue fracture characterized by relatively complex fracture scenarios, including crack bifurcation and crack coalescence events.

- The fatigue interface damage model is able to describe discrete fracture under arbitrary loading conditions, due to the fact that the static interface damage model rigorously accounts for mixed-mode cracking effects.

- The fatigue interface damage model can be applied under variable amplitude loading conditions, whereby load cycle blocks of different amplitude are subsequently imposed in an incremental fashion. 


\begin{tabular}{|c|c|c|c|}
\hline $\mathbf{N}$ & Loading sequence & T-S law & Fracture profile \\
\hline (a) $N=1$ & 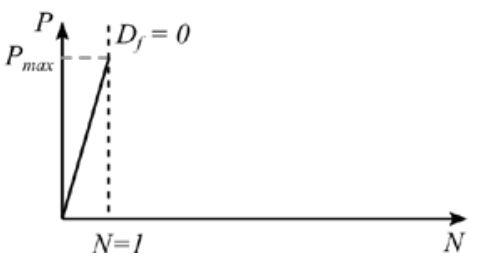 & 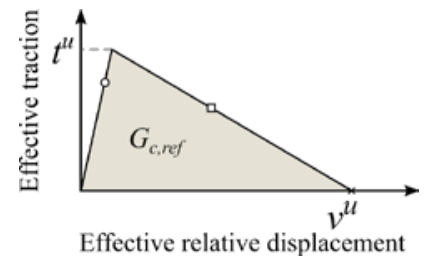 & \\
\hline (b) $N=N 1$ & 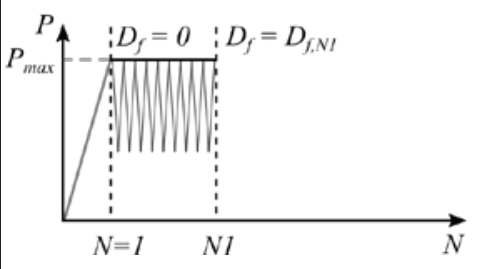 & 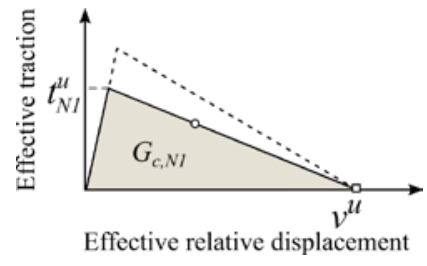 & \\
\hline (c) $N=N 2$ & 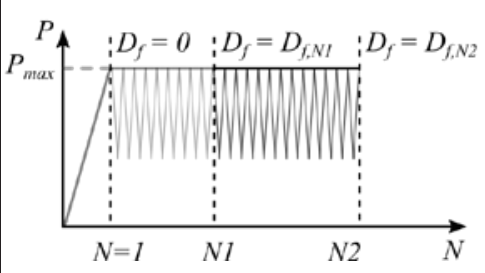 & 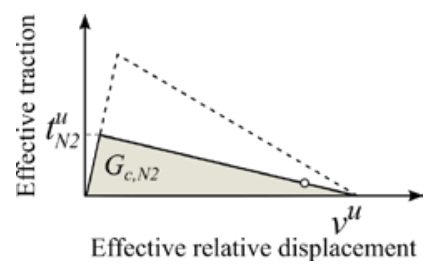 & \\
\hline
\end{tabular}

Fig. 3. Evolution of traction-separation (T-S) law and fracture profile during fatigue loading process. Specific material points in the interface of the double-cantilever beam specimen are indicated by the circle, square and cross symbols.

- The load-cycle driven approach used in the fatigue model is computationally efficient. Instead of describing the precise crack growth in each load cycle, it considers the average crack growth during a number of load cycles. Hence, one load cycle increment may consist of a large number of load cycles, thereby allowing for the economical computation of high-cycle fatigue processes.

\section{Case study}

The discretized equations of the fatigue interface damage model were implemented in a User-defined MATerial (UMAT) model within the commercial finite element program Abaqus. The basic characteristics of the fatigue model are illustrated below by performing a numerical analysis on a relatively simple mode I specimen, see Fig. 4.

\subsection{Problem description}

The specimen shown in Fig. 4 is composed of two continuum elements sandwiching a single interface element. The width and height of the specimen are equal to $10 \mathrm{~mm}$, the thickness of the interface element is set as $0.05 \mathrm{~mm}$, two supports are placed at the bottom nodes of the interface element and a tensile (mode I) fatigue load $P$ is applied at the top of the specimen, which is characterized by a maximum amplitude of $P_{\max }=$ $4.56 \times 10^{3} \mathrm{~N}$ and a load ratio $R=P_{\min } / P_{\max }=0.1$.

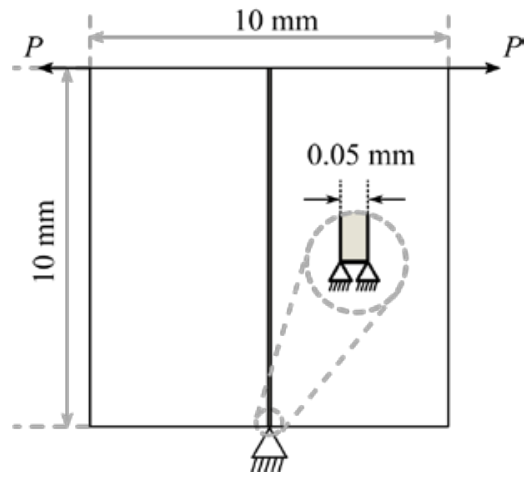

(a) Geometry and boundary conditions.

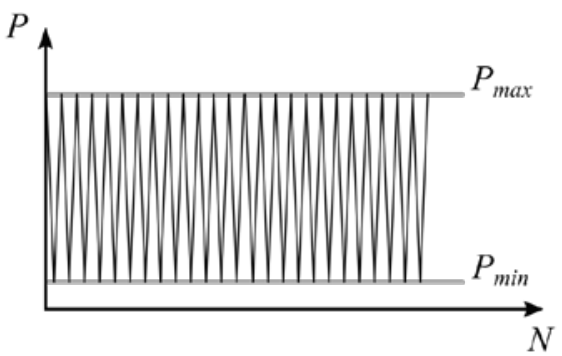

(b) Cyclic loading characteristics.

Fig. 4. Geometry and boundary conditions for a simple mode I specimen load subjected to a tensile fatigue loading $P$ at the top.

The material used for the specimen is a T300/977-2 carbon-fiber reinforced epoxy laminate. The elastic properties are taken from [6] and are listed in Table 1, whereby the "1", "2" and " 3 " material directions correspond to the vertical, horizontal and out-of-plane directions of the specimen. 
Table 1. Elasticity parameters of the specimen.

\begin{tabular}{|c|c|c|c|}
\hline parameter & value & parameter & value \\
\hline$E_{11}$ & $150.0 \mathrm{GPa}$ & $G_{12}$ & $6.0 \mathrm{GPa}$ \\
\hline$E_{22}$ & $11.0 \mathrm{GPa}$ & $G_{13}$ & $6.0 \mathrm{GPa}$ \\
\hline$E_{33}$ & $11.0 \mathrm{GPa}$ & $G_{11}$ & $3.7 \mathrm{GPa}$ \\
\hline$v_{12}$ & 0.25 & $v_{13}$ & 0.5 \\
\hline$v_{23}$ & 0.45 & & \\
\hline
\end{tabular}

The parameters of the interface damage model are listed in Table 2. With these parameters, the static failure load was computed from a preliminary quasi-static analysis as $P_{s}=9.11 \times 10^{3} \mathrm{~N}$. Note that the maximum amplitude of the fatigue loading is half of the static failure load, $P_{\max } / P_{S}=0.5$.

Table 2. Parameters of the interface damage model.

\begin{tabular}{|c|c|c|}
\hline Parameter & Symbol & Value \\
\hline Elastic stiffness & $K$ & $1 \times 10^{6} \mathrm{~N} / \mathrm{mm}^{3}$ \\
\hline Normal strength & $t_{1}^{u}$ & $45 \mathrm{MPa}$ \\
\hline Shear strength & $t_{2}^{u}$ & $45 \mathrm{MPa}$ \\
\hline Mode I fracture toughness & $G_{I, c}$ & $0.268 \mathrm{~kJ} / \mathrm{m}^{2}$ \\
\hline Mode II fracture toughness & $G_{I I, c}$ & $0.632 \mathrm{~kJ} / \mathrm{m}^{2}$ \\
\hline Relaxation parameter & $\eta$ & $2 \times 10^{-6} \mathrm{~s}$ \\
\hline Calibration parameter & $\alpha$ & 90 \\
\hline Calibration parameter & $p$ & 1.2 \\
\hline Deformation threshold & $\lambda_{t h}$ & $2 \times 10^{-5} \mathrm{~mm}$ \\
\hline Calibration parameter & $m$ & 2 \\
\hline Calibration parameter & $n$ & 2 \\
\hline
\end{tabular}

\subsection{Results}

After the load amplitude is imposed on the specimen in a quasi-static fashion, load cycles increments are applied up to the stage at which the specimen fails, which occurs at $N=8990$ load cycles. The deformation profile at fatigue failure is illustrated in Fig. 5, indicating that the fatigue crack has fully developed across the specimen height. The evolutions of the effective damage parameter $D$, the fatigue damage parameter $D_{f}$, the ultimate traction $t^{u}$ and the effective relative displacement $\lambda_{\max }$ in a specific material point of the interface element are illustrated in Fig. 6. It can be observed that the interface element remains elastic during the initial, quasi-static application of the maximum amplitude of the fatigue load, since the effective damage parameter $D$ remains zero during this phase. After this phase has finished $(N=1)$, the specimen is subjected to load cycle increments, during which the fatigue damage parameter $D_{f}$ increases linearly with $N$. As a result of the linear increase, the ultimate traction $t^{u}$ of the tractionseparation curve decreases linearly with $N$, in accordance with Eq.(11). The effective damage $D$ of the interface element starts to develop at $N=7089$. During the subsequent load cycles, the integrity of this element diminishes rapidly, eventually leading to a fully developed crack at $N=8990$, at which $D=1$ and $D_{f}=0.26$. The degradation behavior of the tractionseparation law is illustrated in Fig. 7 by showing this diagram at three different loading stages labeled "1" $(N=1)$, “2” $(N=3589)$ and "3" $(N=7089)$. Stage "1" thus refers to the initial, static traction-separation diagram and stage " 3 " to the traction-separation diagram at fatigue failure, whereby it can be observed that the ultimate mode I traction $t_{1}^{u}$ indeed eventually has been reduced by $D_{f} \times 100 \%=26 \%$, in accordance with Eq.(11).
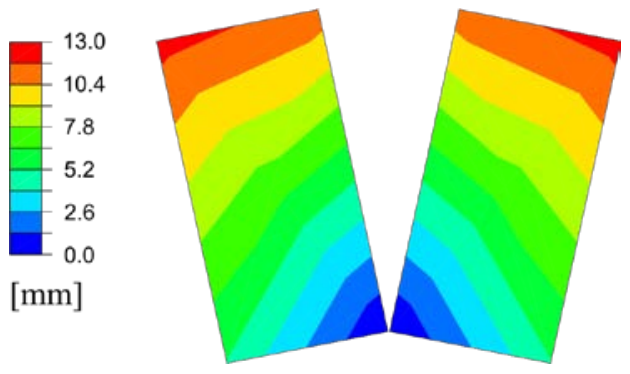

Fig. 5. Deformation profile (not on scale) of mode I specimen at fatigue failure $(N=8990)$.

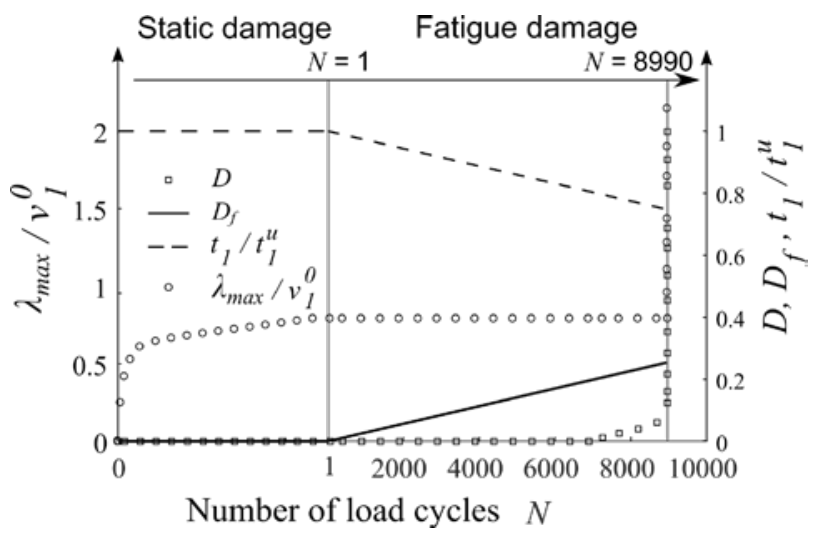

Fig. 6. Effective damage parameter $D$, fatigue damage parameter $D_{f}$, normalized ultimate traction $t^{u} / t_{1}^{u}$ and normalized maximum effective deformation $\lambda_{\max } / v_{1}^{0}$ as a function of the number of load cycles $N$.

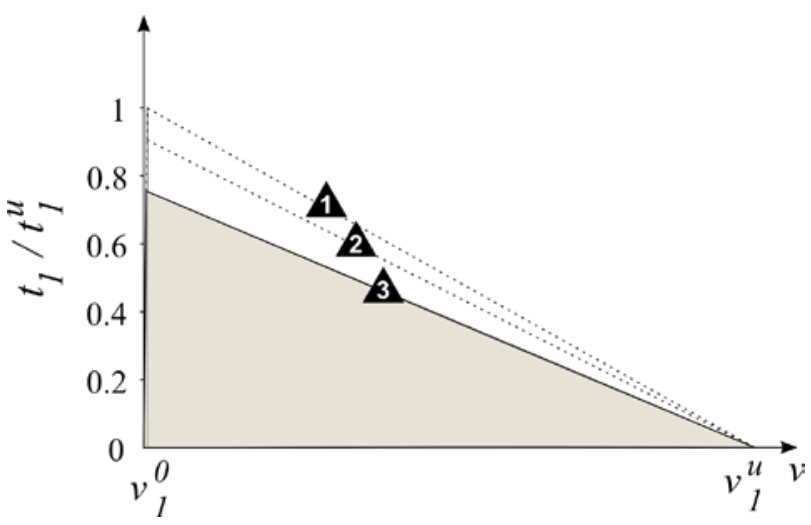

Fig. 7. Traction-separation diagrams at three different loading stages "1" $(N=1)$, "2" $(N=3589)$ and " 3 " $(N=7089)$. 


\section{Conclusions}

In this paper a static interface damage model is combined with a fatigue evolution law to construct a fatigue interface damage model. The fatigue interface damage model can be applied under variable amplitude loading conditions, whereby load cycle blocks of different amplitude are subsequently imposed in an incremental fashion. The model is able to describe discrete fracture under arbitrary loading conditions, due to the fact that the static interface damage model rigorously accounts for mixed-mode cracking effects. Future research will focus on the application of the model for the determination of the fatigue resistance of the blades of wind turbines, whereby complex fracture scenarios, including crack bifurcation and crack coalescence, will be accounted for.

The financial support from the Chinese Scholarship Council (CSC) to F.G is gratefully acknowledged.

\section{References}

1. M.V. Cid Alfaro, A.S.J. Suiker, R. de Borst, J.J.C. Remmers, Eng. Fract. Mech. 76, 761-780 (2009)

2. G. Alfano, M. Crisfield, Int. J. Numer. Meth. Eng. 50, 1707-1736 (2001)

3. P.P. Camanho, C.G. Davila, M.F. De Moura, J. Compos. Mater. 37, 1415-1438 (2003)

4. H. Li, N. Chandra, Int. J. Plast. 19, 849-882 (2003)

5. B.L.V. Bak, C. Sarrado, A. Turon, J. Costa, Appl. Mech. Rev. 66, 060803 (2014)

6. A. Turon, J. Costa, P.P. Camanho, C.G. Dvila, Composites Part A 38, 2270-2282 (2007)

7. P.W. Harper, S.R. Hallett, Int. J. Fatigue 32, 17741787 (2010)

8. A. Pirondi, F. Moroni, J. Adhes. 86, 501-521 (2010)

9. H. Khoramishad, A.D. Crocombe, K.B. Katnam, I. A. Ashcroft, Int. J. Fatigue 32, 1146-1158 (2010)

10. M.H.J.W. Paas, P.J.G. Schreurs, W.A.M. Brekelmans, Int. J. Solids. Struct. 30, 579-599 (1993)

11. R.H.J. Peerlings, W.A.M. Brekelmans, R. de Borst, M.G.D. Geers, Int. J. Numer. Methods Eng. 49, 1547-1569 (2000)

12. H. Khoramishad, A.D. Crocombe, K.B. Katnam, I.A. Ashcroft, Int. J. Adhes. 30, 513-521 (2010)

13. J. Hutchinson, Z. Suo, Adv. Appl. Mech. 29, 63-189 (1992)

14. X.P. Xu, A. Needleman, J. Mech. Phys. Solids. 42, 1397-1434 (1994) 